\title{
Individual and Societal Reactions to Ongoing Terror in Israel
}

\author{
Zeev Wiener \\ Head of community psychiatry programs at "Bril" Community Mental Health Center, \\ Ramat-Chen, Tel-Aviv, Israel. \\ Community Mental Health Center, 9 Hatzvi Street, Tel-Aviv, Israel \\ Correspondence: Dr Weiner zeevw@netvision.net.il
}

Main points:

- Since the beginning of the Al-Aqsa Intifada, belligerent acts have led to the deaths of 244 members of the security forces and 570 civilians; 1,651 members of the security forces and 3,952 civilians sustained injuries.

- Data from Israel and around the world shows that, during the first few days following a terror attack, those directly or indirectly exposed will develop a wide range of symptoms of a depressive and/or anxious nature, which will disappear spontaneously after a few weeks or months.

- A minority at risk will develop Post Traumatic Stress Disorder with accompanying psychiatric morbidity, particularly anxiety and depressive syndromes

- In recent years, Israelis have faced an increasing level of anxiety regarding their personal security, and have become more pessimistic regarding the prospects of securing national security in the wake of the terrorist attacks sustained since September 2000.

As situations that entail death and destruction, war and terror have a profound and enduring impact on individuals and societies. Since September 2000, the conflict that has come to be known as the Al-Aqsa Intifada has raged between Israel and the Palestinians. This conflict is the latest stage in a struggle that has lasted for decades. During the period between September 29, 2000 and July 2, 2003, 18,145 belligerent and terrorist acts occurred in the State of Israel and the Palestinian territories under its control; 733 of these acts occurred in the center of the state. These incidents led to the deaths of 244 members of the security forces and 570 civilians; 1,651 members of the security forces and 3,952 civilians sustained injuries. Most of the deaths and injuries resulted from the activation of incendiary devices or from shooting attacks launched by 
suicide attackers (1). In addition to physical injuries, this protracted conflict has influenced the mental state of individual Israelis and of Israeli society as a whole, with ramifications in the fields of politics, education and the economy - the spheres that form the foundation for individual, societal and national existence.

This article reviews the mental and social ramifications of this situation during the AlAqsa Intifada for the citizens of Israel.

\section{Individual ramifications}

A traumatic event is a sudden incident that deviates from the realms of normal human experience, leading to a sense of horror and a feeling of powerlessness, and involving a significant threat to the victim's psychological or physical existence (2). Several studies have examined the dimensions of this issue including a telephone survey implemented by the Begin-Sadat Institute for Strategic Studies among a representative sample of 500 Israelis, conventional and unconventional attacks by suicide bombers were the commonest cause of anxiety at a significant or greater level among Israelis (3). Shuster (4) undertook a random telephone survey 3-5 days after the terror attack on the World Trade Center among 560 citizens from throughout the United States. He found that $44 \%$ of respondents presented at least one of the following symptoms: A sense of discomfort when something reminded them of the incident; flashbacks, thoughts or dreams relating to the incident; difficulties in concentration or sleep; nervousness; and flashes of anger. The study also found that $98 \%$ of respondents coped with these difficulties by means of conversations with friends, $90 \%$ drew on prayer or spirituality, $75 \%$ checked on their loved ones, and 60\% participated in a group activity. Galea polled 1008 adult residents of Manhattan several weeks after the attacks, and found that the incidence of PTSD was $7.5 \%$ (20\% among residents living close to the WTC). Most people who are exposed to a traumatic event will develop symptoms of anxiety and depression that dissipate after a number of weeks or months (6). A minority will continue to show symptoms as a function of individual and environmental risk factors. Accordingly, it is a logical hypothesis to assume that, after a traumatic event, the majority of those who suffered mental injury will recover (7).

At the end of 2002, Bleich and Solomon (8) carried out a survey among 560 (Israelis. They found that $57 \%$ suffered from bad moods; $55 \%$ stayed away from public places; 
$50 \%$ suffered from sleep disorders; $37 \%$ relived the incident repeatedly; $27.5 \%$ reported disassociation, alienation and temporary memory loss; and $10 \%$ were suffering from fullblown PTSD. In terms of behavioral reactions, $83 \%$ of respondents checked on their loved ones (usually by telephone); $80 \%$ drew support from their family and friends; $74.8 \%$ isolated themselves emotionally; $59.8 \%$ drew strength from religion or other beliefs; and 50.6\% used humor as a coping mechanism. Dolberg (9) examined the occurrence of PTSD among citizens three months after they were examined at a major hospital in downtown Tel Aviv following a terror attack by suicide bombers. Of 129 participants in the survey, 13\% met the criteria for PTSD; $43.5 \%$ showed sub-syndromal disorder; and $43.5 \%$ showed no long-term psychiatric effects. Galili-Weisstub and Lavi examined the occurrence of PTSD among children from various parts of Israel (personal communication). During the peak period of terror attacks, $40 \%$ of children were found to be suffering from PTSD; in small settlements close to Palestinian areas (Gush Katif, Efrat), $20-29 \%$ of children were found to have PTSD. .

In a state of chronic traumatization, the rate of mental morbidity in general, and PTSD in particular, increases, as seen in surveys undertaken in Israel among people examined in emergency rooms following terror attacks and among children. A large number of people in Israel suffer mental trauma due to massive direct and indirect exposure to terror attacks, that demands mental health services reorganization to address treatment to big mass of individuals and communities, for both short-term symptoms and chronic longterm disability. Data from Israel and around the world shows that, during the first few days following a terror attack, those directly or indirectly exposed will develop a wide range of symptoms of a depressive and/or anxious nature, which will disappear spontaneously after a few weeks or months. A minority at risk will develop PTSD with accompanying psychiatric morbidity, particularly anxiety and depressive syndromes. These findings are of great importance in terms of the strategy of operating emergency mental health system. In the first phase following the traumatic event, attention should focus on screening, identifying and rapidly treating the minority of those exposed who are at risk of developing later significant psychopathological disorders, rather than addressing the transient and localized symptoms shown by most of the exposed, and which usually has no significant long-term ramifications. 


\section{Social and national ramifications}

In recent years, Israelis have faced an increasing level of anxiety regarding their personal security, and have become more pessimistic regarding the prospects of securing national security in the wake of the terrorist attacks sustained since September 2000. In $2001,85 \%$ of respondents were concerned that they or those close to them might be injured in a terrorist attack, compared to $58 \%$ in 1999 (10). The same study showed that the surging violence had led to a move to the right among the Israeli public: greater support for military measures to secure an agreement, and support for reducing the points of contact between Israelis and Palestinians. These reactions, which support separatism and the use of force to resolve the conflict, reflect a sense of helplessness expressed in anger and growing anxiety as the result of the increase in terror attacks. The dramatic fall in the number of those who believe it is possible to end the dispute, and the increased assessment that there is a high chance that the war will continue, may suggest that the national mood is one of depression. Prolonged mourning and loss have led to growing pessimism in Israeli society.

Civil violence in the State of Israel, including murder and robbery, is on the increase, as shown in the figures published by the Israel Police. Archer and Gartner (11) compared figures for eighty countries, and found that in those countries involved in wars, there was an increase in murder rates after the war relative to earlier periods. This increase was not found in countries that have not been involved in wars. They found a direct correlation between the number of murders and the number of those injured in wars and terror. In their opinion, acts of killing during war encourage depreciation in the value of human life, legitimize violence and lead violence to be seen as the norm. The security situation, the perceived sense of threat, anxiety, and the use of force to confront terror have seeped through Israeli society, extending the legitimization for the use of force to confront unfamiliar threats. Protracted frustration with the failure to find a solution to the threat of terror has led to anger, expressed in behavioral terms through aggression. Violent communal responses may also be explained by Seligman's learned helplessness model (12), and Milgram's experiments (13) which show that the more an individual feels helpless and loss of control due to intrapsychic factors, the more they are prone to develop anger, anxiety, frustration and depression, expressed in behavioral terms as violence. 
In a survey carried out among a representative sample of 509 Israeli Jews, $54.8 \%$ were very or extremely optimistic regarding Israel's future, and only $18.6 \%$ defined themselves as pessimistic or very pessimistic (14). This finding contrasts with the finding in the same survey that $42.8 \%$ of respondents feel very or extremely powerless, and that this feeling has increased among over $50 \%$ of respondents since the events of September 2000.

These findings show that the collective Israeli psychological status includes despair and powerlessness, but paradoxically also optimism and hope. This is a communal dissociative phenomenon typical to chronic traumatization. It is responsible also to the widening socio-economic gaps in the Israeli society resulting reduced emotional availability to the distress faced by others, and withdrawal into an individual or sectarian psychological world. Dissociation may serves as a defense mechanism against shearing forces of trauma, to enable surviving in the acute state. Yet, being chronic, it mitigates against dialogue and the effort to reach an agreement with the other side by jeopardizing reality judgment.

Despite protracted and severe terrorism, numerous wars, missile attacks on population centers in the center of the country and a constant threat from its neighbors, the national infrastructure has not collapsed and Israel continues to exist as an independent and democratic state. A survey of Jewish adolescents living in communities in the Palestinian territories(15) found that the level of anxiety was intermediate or below, despite the protracted and high level of exposure to terror attacks, injuries, death and destruction. The decline in anxiety levels reflects a considerable measure of habituation, which is also reflected in the fact that, after serious terror attacks, life continues as normal, including visits to cafes and the use of public buses - two of the main targets for suicide bombers.

The national Israeli response to terror is also influenced by factors constituting a source of cohesion and providing a sense of control and support. These factors among others include: scientific achievements, a basic common Jewish identity; an emerging Israeli identity, based mainly on language and culture; freedom of expression and association based on the democratic infrastructure of the State of Israel. Terror is a pathogenic factor, yet, it encourages communal cohesion and leads to the suspension of internal disputes in the face of the common threat. 


\section{Systemic therapeutic perception}

Traumatic incidents are incidents of a flooding nature that are associated with significant losses and foster a complex psychiatric, psychological and social clinical picture with long-term effects on individuals and communities. Studies have shown that mental health intervention can help reduce psychological morbidity and behavioral changes (16). Regrettably, most of the programs tend not to be systemic, focusing on individuals or groups on a short- or medium-term basis, giving insufficient attention to prevention and inoculation, and lacking a base in a single comprehensive theory of how to cope with disasters in general, and protracted trauma in particular. In order to integrate the needs of individuals and communities subject to protracted threat, and with the objective of preventing morbidity, encouraging mental resilience and ensuring effective treatment for victims, a comprehensive and socially oriented approach must be adopted (17). Such an approach mandates the establishment of well-developed public mental health services, closely integrated with other institutional and non-institutional bodies, such as: national and local welfare services, the police and army, primary care medicine, general and psychiatric hospitals, education systems and voluntary organizations. There is a need to redefine and broaden the functions of the relevant professions. Therapeutic teams must develop the capability to act in cooperation with additional disciplines and systems with different organizational languages, and to cope with such technical and organizational obstacles as limited resources, organizational problems, difficulties in communication and the absence of real-time information (18).

Laor (19) has proposed a comprehensive theory for coping with disaster based on principles drawn from the fields of public health, preventative medicine, social work, education and urban planning. His theory outlines an infrastructure from which a model may be drawn for a multiprofessional and intersystemic community mental health service for bio-psychosocial and educational interventions. This model is designed to respond to the reactions of individuals and communities to protracted trauma and disaster events while ensuring maximum synchronization - a critical factor in coping with trauma. The model enables a proper evaluation of damage, the implementation of broad-based interventions as required, the monitoring of changing needs, the preparation of the population in order to enhance their mental coping with future events, and the maintenance of functions on the individual and community levels. This model began to crystallize in 1991, when Israel was subject to dozens of Iraqi missile attacks. Since 
then, given the massive wave of terror attacks in recent years, the model has continued to be applied and developed in Tel Aviv, through the Cohen Harris Trauma and Disaster Center and has been adopted by local and governmental authorities around Israel.

Since the events of September 11 in the United States, there has been growing recognition of the need to cope with terror. The world is a global village facing a more tangible threat than ever before. The ramifications of this threat are not only local, and the challenge faces not merely one or more particular communities, but the entire free world. Mutual learning from the sad experience of terror-struck regions, and international cooperation in organizing mental health services and treating the population, will help reduce the destructive impact of terror acts, save lives, reduce mental morbidity and suffering, and maintain hope for a better world.

\section{References}

1. IDF Website: http://www.idf.il

2. American Psychiatric Association. Diagnostic and Statistical Manual of Mental Disorders, Fourth Edition, Washington, DC: American Psychiatric Association 1995.

3. Ventura R. Terrorism and public opinion. Begin-Saadat Center for Strategic Studies and International Policy Institute for Counter Terrorism 1999; http://www.biu.ac.il

4. Schelenger WE et al. Psychological reactions to terrorist attack. Finding from the national study of American's reactions to September 11. JAMA 2002; 288(5): 581-88.

5. Galea $S$ et al. Psychological sequelae of the September 11 terrorist attacks in New York City. N Eng J Med 2002; 346(13): 982-87.

6. Smith T, Rasinski K and Toce M. America rebounds: a national study of public response to the September 11th terrorist attacks. A National Organization for Research at the University of Chicago. 2001; http://www.norc.uchicago.edu/

7. National Institute of Mental Health 2002. Mental health and mass violence: evidence based early psychological intervention for victims' survivors of mass violence. A workshop to reach consensus on best practices. NIH publication No. 02-5138, Washington, DC: US government printing office.

8. Bleich A. The struggle of the Israeli society with terror and trauma. Presented at the Israeli Psychiatric Association Annual Meeting, Haifa, April 2003. Since this article was first written Dr Bleich's presentation has been published as follows: Bleich A, 
Gelkopf M, Solomon Z. Exposure to terrorism, stress-related mental health symptoms, and coping behaviors among a nationally representative sample in Israel. JAMA. 2003 Aug 6; 290(5): 612-20

9. Dolberg OT et al. The prevalence of PTSD among survivors of terror attacks-a report of 129 cases. Presented at the Israeli Psychiatric Association Annual Meeting, Haifa, April 2003.

10. Arian A. Israeli public opinion on national security. Jaffee Center for Strategic Studies, Tel Aviv University 2001: Memorandum No. 60.

11. Archer D and Gartner R. Violence and crime in cross-national perspective, New Haven: Yale University Press 1984.

12. Seligman M. Learned helplessness. Annu Rev Med 1972; 23:407-12.

13. Milgram NA. Psychological stress and adjustment in time of war and peace: the Israeli experience as presented in tow conferences. Isr Ann Psychiatr Relat Discip 1978; 16(4): 327-38.

14. Zakai D. Depressed, helpless and reluctant to learn. Panim Quarterly for Society, Culture and Education 2001; 17:4-11.

15. Kalish N. A moment before we go insane. Panim Quarterly for Society, Culture and Education 2001; 17:12-22.

16. Breslau N. Outcomes of posttraumatic stress disorder. J Clin Psychiatry. 2001;62 Suppl 17:55-9.

17. Laor N. The open and closed society from the perspective of disaster situation. Presented at the Centennial Popper's Conference. Vienna, July 2002.

18. Laor N. The role of mental health professionals after mass disaster. Presented at the Promised Childhood Congress. Tel-Aviv, May 2001.

19. Laor $\mathrm{N}$ et al. Facing War, terrorism and disaster: towards a child- oriented comprehensive emergency care system. Child Adolescent Psychiatric Clin N Am 2003; 12;343-61. 